\title{
Programmed Cell Death 1 Receptor / Programmed Death-Ligand1 (PD-1/PD-L1 ) Inhibitors Alone or with Conventional Therapy versus Standard of Care for Head and Neck Cancer: A Systematic Review and Meta-Analysis
}

\author{
Xin Huang ${ }^{1}$, Jie Zhang ${ }^{2}$, and Hongying Ruan ${ }^{3}$ \\ ${ }^{1}$ Tianjin Medical University \\ ${ }^{2}$ Huainan Union University \\ ${ }^{3}$ Tianjin First Central Hospital
}

June 14, 2021

\begin{abstract}
Objectives As a pivotal part of precision therapy, PD-1/PD-L1 immunotherapy has been gradually used in head and neck cancer (HNC). We investigated the effect and adverse events of PD-1/PD-L1 inhibitors alone or with conventional therapy. Design The groups using PD-1/PD-L1 immunotherapy or combining with conventional therapy were defined as the experimental groups, while the standard of care were the control groups. Cochrane Library, Embase, PubMed, and Web of Science were undertaken to identify literature up to November 20, 2020. Overall survival (OS) and progression-free survival (PFS) were the primary outcome measures. Secondary outcome measures included objective response rate (ORR), disease control rate (DCR), any grade and grade[?]3 adverse events (AE). Results Five randomized controlled trials (RCTs) and nine single-arm trials were included in the systematic review. The OS of the experimental groups was better than the control groups $(\mathrm{OR}=$ 0.63, 95\% CI: $\left.0.49-0.82, \mathrm{I}^{2}=35 \%, \mathrm{P}=0.0004\right)$, particularly in patients who smoke current/former or with human papillomavirus negative (HPV-). The experimental groups had longer OS than the control groups in patients with PD-L1 positive $(\mathrm{OR}=0.75$, 95\% CI: $0.65-0.85, \mathrm{I} 2=0 \%, \mathrm{P}<0.0001)$. Patients with HPV positive $(\mathrm{HPV}+)$ had more favorable OS than those with HPV- (OR $=0.56,95 \%$ CI:0.44-0.71, I2=39\%, P<0.00001). There were statistical differences in AEs (e.g., fatigue, rash, hypothyroidism, etc.) and no statistical differences in PFS, ORR, and DCR. Conclusion PD-1/PD-L1 immunotherapy or combining with conventional therapy can improve the treatment effect and induce fewer adverse events of digestion and blood system, except for hypothyroidism.
\end{abstract}

Programmed Cell Death 1 Receptor / Programmed Death-Ligand1 (PD-1/PD-L1 ) Inhibitors Alone or with Conventional Therapy versusStandard of Care for Head and Neck Cancer: A Systematic Review and Meta-Analysis

\section{Abstract :}

ObjectivesAs a pivotal part of precision therapy, PD-1/PD-L1 immunotherapy has been gradually used in head and neck cancer (HNC). We investigated the effect and adverse events of PD-1/PD-L1 inhibitors alone or with conventional therapy.

Design The groups using PD-1/PD-L1 immunotherapy or combining with conventional therapy were defined as the experimental groups, while the standard of care were the control groups. Cochrane Library, Embase, PubMed, and Web of Science were undertaken to identify literature up to November 20, 2020. Overall survival (OS) and progression-free survival (PFS) were the primary outcome measures. Secondary outcome measures 
included objective response rate (ORR), disease control rate (DCR), any grade and grade[?]3 adverse events (AE).

Results Five randomized controlled trials (RCTs) and nine single-arm trials were included in the systematic review. The OS of the experimental groups was better than the control groups (OR $=0.63,95 \%$ CI: 0.49$0.82, \mathrm{I}^{2}=35 \%, \mathrm{P}=0.0004$ ), particularly in patients who smoke current/former or with human papillomavirus negative $\left(\mathrm{HPV}^{-}\right)$. The experimental groups had longer OS than the control groups in patients with PDL1 positive $(\mathrm{OR}=0.75,95 \% \mathrm{CI}: 0.65-0.85, \mathrm{I} 2=0 \%, \mathrm{P}<0.0001)$. Patients with HPV positive $\left(\mathrm{HPV}^{+}\right)$had more favorable OS than those with $\mathrm{HPV}^{-}(\mathrm{OR}=0.56,95 \% \mathrm{CI}: 0.44-0.71, \mathrm{I} 2=39 \%, \mathrm{P}<0.00001)$. There were statistical differences in AEs (e.g., fatigue, rash, hypothyroidism, etc.) and no statistical differences in PFS, ORR, and DCR.

Conclusion PD-1/PD-L1 immunotherapy or combining with conventional therapy can improve the treatment effect and induce fewer adverse events of digestion and blood system, except for hypothyroidism.

Key points PD-1/PD-L1 inhibitors alone or with conventional therapy can improve OS; patients with PD-L1 combined positive score (CPS) >1 were more suitable for PD-1/PD-L1 inhibitors, and HPV positive patients had a more favorable OS; higher risk of hypothyroidism in PD-1/PD-L1 inhibitors; OS of patients with HPV negative and smoke current/former in PD-1/PD-L1 inhibitors are better than Standard of Care; Fewer adverse events of PD-1/PD-L1 inhibitors both any grade and grade[?]3 (e.g., nausea, decreased appetite, anemia, etc.).

\section{PROSPERO (CRD42020223177)}

Keywords : head and neck cancer, immune checkpoint inhibitors, Programmed Cell Death 1 Receptor (PD-1), Programmed Death-Ligand1 (PD-L1), human papillomavirus, immunotherapy

\section{Introduction}

As the seventh most common tumor (1), head and neck cancer (HNC) consists of four major anatomical sites: the oral cavity, oropharynx, larynx, and nasopharynx (2), and almost $90 \%$ of it is head and neck squamous cell carcinoma (HNSCC)(3). HNSCC associates with the human papillomavirus (HPV) (HPV16/18) and tobacco (4), and the overall incidence rate of HNSCC in males is higher than in females (4). HPV-positive $\left(\mathrm{HPV}^{+}\right)$patients have a more favorable prognosis and more favorable responses to chemotherapy and radiotherapy than patients with $\mathrm{HPV}$-negative $\left(\mathrm{HPV}^{-}\right)(5)$. Conventional treatments for $\mathrm{HNC}$ involve surgery and chemoradiotherapy. Cetuximab, the antibody of epidermal growth factor receptor (EGFR), is also applied to the HNC treatment combined with cisplatin-based chemotherapy $(6,7)$. However, the efficiency of conventional treatments and cetuximab is limited(8). One of the first and most characterized immune evasion mechanisms is the PD pathway (9). Overwhelming evidence demonstrated that PD-1/PD-L1 immune inhibitors could reduce escaping tumor immune surveillance ( $T$ cell suppression)(10), enhance endogenous anti-tumor immunity (11). We first identified the suitable population for PD-1/PD-L1 inhibitors, compared the overall survival rate of the patients with different characteristics, analyzed the incidence of various adverse events in detail according to the classification, and included studies about PD-1/PD-L1 inhibitors with conventional therapy to guide clinical practice, which has not been studied(12-14).

\section{Methods}

Protocol and registration

The systematic review and meta-analysis was conducted under the principles of the Preferred Reporting Items for Systematic Reviews and Meta-Analyses (PRISMA) 2009 checklist (15) and registered at Prospective Register of Systematic Reviews (PROSPERO) (CRD42020223177). Tianjin First Central Hospital Research Ethics Committee had confirmed that no ethical approval was required.

Eligibility criteria 
We screened literature following PICOS questions. The population included patients with HNC; the intervention included treatment either PD-1/PD-L1 immunotherapy alone or combining conventional therapies (e.g., chemotherapy, radiotherapy, and cetuximab); the comparison included standard-of-care therapy; the outcome included OS, PFS, AE, ORR, and DCR; the study design included RCTs and prospective single-arm clinical trials. The language type was English, and the types of articles excluded were conference abstracts, reviews, letters, personal opinions, book chapters, trial protocols, systematic reviews and meta-analyses because of the lack of complete and reliable data. Publications that reported duplicated data from the same clinical trials were also excluded, and the most complete of them were included.

Information sources, search, and study selection

We searched in the electronic databases Cochrane Library, Embase, PubMed, and Web of Science until November 20, 2020. The search strategies were uploaded in Supplementary File 1 . Duplicate references were removed with reference manager software (EndNote(r)), and the rest of the references were personally screened. We performed the first screen based on titles and abstracts and excluded the references that do not meet the eligibility criteria after getting access to full-text articles on the second screen.

Data extraction

Data from full-text articles were extracted by us independently. Study characteristics (the trial name, publication year, phase of studies), baseline characteristics of patient populations (amount of participates, amount and proportion of males, age, HPV, smoke status, Eastern Cooperative Oncology Group (ECOG) performance status score), study design (type and dosage of PD-1/PD-L1 immune inhibitors), outcomes (OS, PFS, ORR, DCR, the median time to response, the median duration of follow-up and response,) and amount of any-grade and grade [?]3 AEs in the articles were collected from individual studies.

Data synthesis and statistical analysis

OS and PFS were the primary outcomes, while the secondary outcomes included ORR, DCR, any grade, and grade[?]3 AE. Survival data from the figures in the references were extracted by Engauge Digitizer 4.1 software(16), and $\ln [$ Hazard Ratio] $(\ln [\mathrm{HR}])$ and $\operatorname{Seln}[\mathrm{HR}]$ of survival curves were calculated by the Microsoft Excel program file(17). Cochrane Collaboration's Review Manager(r)5.4 software (RevMan5.4) was used to conducted meta-analyses. Inconsistency indices (I2) were used to test the studies' substantial amount of heterogeneity; fixed-effect models were performed when I2[?] $50 \%$. Otherwise, heterogeneity was considered among the studies, either through sensitivity analysis or subgroup analysis to explore heterogeneous sources, and random effect models were performed after eliminating evident clinical and methodological heterogeneity. Summary measure was the odds ratio $(\mathrm{OR}) . \mathrm{P}<0.05$ indicated a statistically significant difference.

Risk of bias

The risk of bias of individual studies was assessed by the Cochrane collaboration's Risk of Bias Tool based on the following seven domains: selection bias, selection bias, performance bias, detection bias, attrition bias, reporting bias, and other biases. Each risk of bias was defined as low, unclear, or high by Xin Huang and Jie Zhang independently.

The quality of evidence and the strengths of recommendations were evaluated following the Grading of Recommendation, Assessment, Development, and Evaluation (GRADE) guidelines $(18,19)$ through informatics tools from GRADEpro (20) based on study design, risk of bias, inconsistency, indirectness, imprecision, and other considerations. Egger's test evaluated latent publication bias in Stata 16 software.

\section{Results}

Study selection and characteristics

We obtained 862 articles after searching through databases, and 759 articles remained after removing duplicates. We excluded 601 articles according to the titles and abstracts, then got 158 full-text articles, and finally included five RCTs and nine single-arm trials in this review (Figure 1 )(15, 21-34). 
Three of five RCTs used PD-1/PD-L1 immune inhibitors alone, and one combined with conventional therapy. Experimental groups used both individual and combination therapies In one of the $\operatorname{RCTs}(22)$. Six of nine single-arm trials used PD-1/PD-L1 immunotherapy alone, and three combined with conventional therapy.

Our study included five phase-I clinical trials, one phase I/II trials, four phase II trials, and four phaseIII trials. This review included 2,911 patients with HNC, in which control groups contained 959 patients. Experimental groups contained 1350 patients in the 5 RCTs, in which 1028 patients were treated with PD-1/PD-L1 immune inhibitors alone, and 322 patients were treated with PD-1/PD-L1 immunotherapy combining conventional therapy; while 602 patients in the nine single-arm trials, in which 504 participants were treated with PD-1/PD-L1 inhibitors alone and 98 participants were treated with PD-1/PD-L1 inhibitors with conventional therapy. The anti-PD-1/PD-L1 mAbs used in the experimental groups included nivolumab $(\mathrm{n}=1)$, pembrolizumab $(\mathrm{n}=6)$, durvalumab $(\mathrm{n}=4)$, avelumab $(\mathrm{n}=2)$, and atezolizumab $(\mathrm{n}=1)$. The characteristics of studies and primary outcomes were presented inTable $\mathbf{1}$ and Supplementary File $\mathbf{2}$, respectively.

\section{OS, PFS, and PD-L1 related OS}

Available data of overall survival were extracted from $3(21,23,24)$ of 5 RCTs and represented experimental groups significantly improves overall survival $(\mathrm{OR}=0.63,95 \% \mathrm{CI}$ : $0.49-0.82, \mathrm{I} 2=35 \%, \mathrm{P}=0.0004)$ (Figure 2a) while the data from $2(21,23)$ of 5 RCTs showed that there was no statistical difference in progression $(\mathrm{OR}=0.69,95 \%$ CI: 0.48-1.01, I2 $=0 \%, \mathrm{P}=0.05)$ (Figure $\mathbf{2 b})$. Notably, the risk of death was lower in the experimental group than in the control group in participants with HPV negative $(\mathrm{OR}=0.54,95 \%$ CI: $0.36-0.81, \mathrm{I} 2=0 \%, \mathrm{P}=0.003)$ and smoke current/former (OR $=0.64,95 \%$ CI: $0.45-0.91$, I2 $=2 \%$, $\mathrm{P}=0.01$ ), whereas the patients with other factors (e.g., female, ECOG=0, ECOG[?]1, smoke never, and HPV positive) did not show statistically significant differences in overall survival (Supplementary File 3 ).

PD-L1 status was characterized by PD-L1 combined positive score (CPS) calculated by the percentage of tumor cells, lymphocytes, and macrophages in the amount of tumor cells (22, 23). PD-L1 was positive when PD-L1 CPS [?]1, otherwise PD-L1 was negative. In PD-L1 positive patients, experimental groups survived longer than control groups ( $\mathrm{OR}=0.75,95 \% \mathrm{CI}: 0.65-0.85, \mathrm{I} 2=0 \%, \mathrm{P}<0.0001)$ (Figure 2c ) while no statistical difference was found between the two groups in PD-L1 negative patients (OR $=0.96,95 \%$ CI: $0.71-1.30, \mathrm{I} 2=0 \%, \mathrm{P}=0.79)($ Figure 2d $)$.

\section{HPV related OS, ORR and DCR}

$\mathrm{HPV}^{+}$patients had a more favorable OS than HPV ${ }^{-}$patients in PD-1/PD-L1 immune inhibitors alone or with conventional therapy ( $\mathrm{OR}=0.56,95 \%$ CI: 0.44-0.71, I2 $=39 \%, \mathrm{P}<0.00001)$ (Figure 3a ). ORR $(\mathrm{OR}=1.66,95 \% \mathrm{CI}: 1.01-2.73, \mathrm{I} 2=0 \%, \mathrm{P}=0.05)($ Figure $3 \mathrm{~b})$ and $\mathrm{DCR}(\mathrm{OR}=0.92,95 \% \mathrm{CI}$ : $0.55-1.56$, $\mathrm{I} 2=29 \%, \mathrm{P}=0.77)($ Figure 3c $)$ showed no significant differences between HPV positive patients and HPV negative patients.

Treatment-related AEs

the common types of treatment-related AEs

The most common type of any grade treatment-related AEs in PD-1 inhibitors groups was fatigue (18.76\%, 95\% CI (\%): 16.35- 21.16), while grade[?]3 AEs was anemia (2.07\%, 95\% CI (\%): 1.20- 2.95). In PD-L1 inhibitors groups, the most common type of any grade AEs was also fatigue (11.22\%, 95\% CI (\%): 8.48 13.97) (Online Resource 2), while grade[?]3 AEs was Gamma-glutamyl transferase (GGT) increased (1.18\%, 95\% CI (\%): 0.24- 2.12). The most common type of any grade AEs in PD-L1 inhibitors with conventional therapy groups was called mucositis oral $(96.08 \%, 95 \%$ CI (\%): 90.75- 100.00), which was the most common grade[?]3 AEs in the groups as well (52.94\%, 95\% CI (\%): 39.24- 66.64). All the common types of treatmentrelated AEs are given inSupplementary File 4 .

Any grade AEs and grade[?]3 AEs 
The occurrences of any grade AEs in participants treated with PD-1/PD-L1 immunotherapy alone were much lower than standard of care $(\mathrm{OR}=0.34,95 \% \mathrm{CI}: 0.26-0.44, \mathrm{I} 2=0 \%, \mathrm{P}<0.00001)$ (Figure 4a $)$. The occurrences of grade[?]3 AEs in PD-1/PD-L1 immunotherapy alone groups were much lower than standard of care groups $(\mathrm{OR}=0.30,95 \% \mathrm{CI}: 0.22-0.40, \mathrm{I} 2=0 \%, \mathrm{P}<0.00001)($ Figure $4 \mathrm{~b})$.

Individual analyses of treatment-related AEs

In the PD-1/PD-L immunotherapy alone groups, the risk of some categories of any grade AEs (fatigue, nausea, decreased appetite, anemia, asthenia, vomit, stomatitis, weight loss, mucosal inflammation, alopecia, neutropenia, dermatitis acneiform, leukopenia, and neuropathy peripheral) were lower than control groups, which revealed PD-1/PD-L1 inhibitors might induce less harm in the digestive system and the blood system than standard of care. However, the incidence of hypothyroidism was higher in the experimental groups $(\mathrm{OR}=26.88,95 \%$ CI: 7.54-95.86, I2 $=0 \%, \mathrm{P}<0.00001)$. The incidence of any grade of diarrhea had no significant statistical differences between experimental and control groups (Figure $\mathbf{5}$ ). Some types of grade[?]3 AEs (e.g., nausea, rash, decreased appetite, anemia, asthenia, stomatitis, mucosal inflammation, neutropenia, neutrophil count decreased, and leukopenia) occurred less in experimental groups. In contrast, no statistically significant differences were observed in grade[?]3 AEs (e.g., fatigue, and diarrhea) between the two groups (Figure 6 ).

In the only using PD-1 inhibitors experimental groups, the incidence of some categories of any grade AEs (e.g., fatigue, anemia, asthenia, vomit, stomatitis, weight loss, mucosal inflammation, alopecia, neutropenia, and nausea) were lower than control groups. In contrast, the incidence of any grade of diarrhea in PD-1 inhibitors experimental groups had no significant statistical differences compared to control groups (Supplementary File 5 ). There were fewer grade [?]3 AEs (e.g., anemia, asthenia, stomatitis, mucosal inflammation, neutropenia, neutrophil count decreased, rash, and nausea) in the only using PD-1 inhibitors experimental groups except for fatigue and diarrhea (Supplementary File 6 ). In contrast, the incidence of grade [?]3 of fatigue in PD-1 inhibitors experimental groups had no significant statistical differences compared to control groups.

\section{Risk of bias}

Risk of bias within studies

The risk of bias within the five randomized controlled trials was evaluated (Supplementary File 7 ) by the bias risk assessment tool provided in the Cochrane Evaluation Manual, and 30 items were defined as low risk, 11 items were unclear, and only eight items were defined as high risk, while the risk of bias within the nine single-arm studies could not be evaluated. All the studies were open-label trials because of the inevitable occurrences of treatment-related adverse events.

\section{Publication bias}

Publication bias of OS, ORR, DCR, clinical characteristics subgroups analyses of OS with HPV positive or HPV negative, any grade and grade [?]3 AE calculated by Egger's test in Stata 16 software were 0.384, $0.679,0.248,0.849,0.965,0.704,0.667$, respectively. Publication bias of any grade AEs of PD-1/PD-L1 inhibitors, any grade AEs of PD-1 inhibitors, grade [?] 3 AEs of PD-1/PD-L1 inhibitors, grade [?]3 AEs of PD-1 inhibitors were calculated by Egger's Test, as shown in Supplementary File 8 .

The quality of evidence

The quality of evidence from 27 outcomes across studies was evaluated as high, 21outcomes' were moderate by GRADE system (20), which were shown in Supplementary File 9 .

\section{Discussion}

The majority of HNC was diagnosed at stage III-IV, and its high fatality rate and poor quality of life become significant concerns to social and public health (35). HPV, heavy use of tobacco and alcohol can increase the 
risk of $\mathrm{HNC}(36)$, which has been proved by various studies. Recent studies have witnessed the development of immunotherapy of HNC. PD-1 was first discovered and associated with suppressing T cells, downregulating the immune response, and apoptosis(37). PD-L1(38), one of the ligands of PD-1, is expressed in T cells and antigen-presenting cells; PD-L2(39), another ligand of PD-1, is present in antigen-presenting cells, stromal, tumor endothelial cells, can be induced by other cells as well(40).

To investigate the effect of PD-1/PD-L1 inhibitors alone or in combination with traditional therapy, we performed the systematic review and meta-analysis included five RCTs and nine single-arm clinical trials, which contained 2,911 HNC patients. The 5 RCTs revealed that immunotherapy improved survival rates by $63 \%$ compared with SOC. The reasons we inferred are as follows, HNC is characterized by inadequate immune surveillance, more immunosuppressive cytokines, and impairment of immune cells both in function and number(41); immunotherapy may enhance the capacity of the immunity system to inhibit tumor cell infiltration. However, DNA repair defects associated with migratory and invasive behavior may induce the poor prognosis of HNC patients treated with chemotherapy may induce (42). Of note, conventional therapies are lack specificity.

We observed patients with HPV ${ }^{+}$had better overall survival $(\mathrm{P}<0.000001)$, and participants with PD-L1 CPS [?]1 were more suitable for PD-1/PD-L1 immunotherapy $(\mathrm{P}<0.0001)$. Viral antigens exist as exosomes or integrate into host cells throughout carcinogenesis (43), which may strengthen the immunity of $\mathrm{HPV}^{+}$ patients (44). $\mathrm{HPV}^{+} \mathrm{HNSCC}$ enriched more B cells (44), and PD-1 expression was lower in CD8 ${ }^{+} \mathrm{T}$ cells, while the PD-1 frequency negatively correlated with IFN- $\gamma$ secretion capacity (45). The frequency of PD-1 positive tumor-infiltrating lymphocytes (TIL) was higher in $\mathrm{HPV}^{+}$patients, but their frequency of $\mathrm{CD}^{+}$ regulatory $\mathrm{T}$ cells (Tregs) cells was slightly lower than HPV-TILs(44). The enrichment area of B cells in $\mathrm{HPV}^{+}$TIL was found primarily in the germinal center, in which B cells experienced germinal center reactions and could appear at different stages of development; while the majority of B cells in HPV TILs $^{-}$ is mainly plasma cells and switched memory cells (44). Additionally, TP53 mutations (46), amplification, and overexpression of CCND1(the gene that encodes Cyclin D1) induced drug resistance, which was more common on $\mathrm{HPV}^{-}$tumors (47).

PD-L1 overexpression was considered to influence prognosis of HNSCC(48). PD-L1 on exosomes was associated with disease progression of HNSCC patients, including patients' disease activity and the lymph node status(49). Overexpression PD-L1 on circulating tumor cells (CTC) indicated worse OS and PFS at the end of treatment for locally advanced $\operatorname{HNSCC}(50)$. In contrast, it was relevant to favorable survival when overexpression on tumor cells together with tumor-associated immune cells (TAICs)(47), which seems to be paradoxical and needs more investigation. Besides, some studies revealed that PD-L2 expression was the potential prognostic biomarker of PFS and response to pembrolizumab in HNSCC patients (40), PD-L1 and IFN- $\gamma$ related gene signatures impacted effects of PD1/PD-L1 immunotherapy in recurrent or metastatic HNSCC(47). Intriguingly, patients with high PD-1 expression also have a better response to PD-1 inhibitors(45).

Although PD-1/PD-L1 immunotherapy influences less on the digestive system and blood system, it might cause a higher risk of hypothyroidism. In a report about nivolumab-related thyroiditis, some of the patients endured transient thyrotoxic phases followed by thyroiditis, part of them with antithyroglobulin (anti-Tg) and antithyroid peroxidase (TPO) antibodies. However, thyrotropin binding inhibitory immunoglobulins (TBII) of all of them were absent, while the other patients developed hypothyroidism without the thyrotoxic phase, anti-Tg, and anti-TPO antibodies were positive(51). Some patients with hypothyroidism but without thyrotoxicosis might endure dormant thyrotoxic stage, and the mechanism for PD-1/PD-L1 immunotherapy related-thyroid dysfunction was unclear(52). We assumed PD-1/PD-L1 immunotherapy induced thyroiditis, thus caused hypothyroidism and the most common fatigue symptoms (52). In a nutshell, it is necessary to test thyroid function after receiving PD-1/PD-L1 immunotherapy.

\section{Limitations}


Only five RCTs and two RCTs referring to PD-1/PD-L1 antibodies combining with other therapy we collected limited the analysis. All the RCTs were open-label trials, which might influence compliance and detection bias. We also included some single-arm trials, whereas some had small numbers of participants, especially in the trials using combination therapy. Publication bias of PFS, subgroups analyses of OS with clinical characteristics (female, smoke current/former, never smoke, ECOG=0, and ECOG[?]1) some types of any grade AEs of PD-1/PD-L1 inhibitors (e.g., vomit, weight loss, dermatitis acneiform, leukopenia, neuropathy peripheral and hypothyroidism), grade [?]3 AEs of PD-1/PD-L1 inhibitors (e.g., neutrophil count decreased, decreased appetite, and leukopenia), any grade AEs of PD-1 inhibitors (e.g., vomit, weight loss, alopecia, neutropenia, nausea, and diarrhea), and grade [?]3 AEs of PD-1 inhibitors (neutrophil count decreased) could not be detected because of the limited number of available studies. However, the occurrence of any grade hypothyroidism in PD-1/PD-L1 inhibitors was much higher than standard of care, which could not be overlooked.

\section{Conclusions}

PD-1/PD-L1 immune antibodies alone or with conventional treatments may prolong the OS of participants with HNC and caused fewer AEs except for hypothyroidism. Besides, patients with HPV positive and or PD-L1 CPS[?]1 had a lower risk of death, while no statistical differences in ORR and DCR were observed between different HPV status and experimental groups and control groups.

\section{References}

1. Bray F, Ferlay J, Soerjomataram I, Siegel RL, Torre LA, Jemal A. Global cancer statistics 2018: GLOBOCAN estimates of incidence and mortality worldwide for 36 cancers in 185 countries. CA: a cancer journal for clinicians. 2018;68(6):394-424.

2. Lydiatt WM, Patel SG, O’Sullivan B, Brandwein MS, Ridge JA, Migliacci JC, et al. Head and Neck cancers-major changes in the American Joint Committee on cancer eighth edition cancer staging manual. CA: a cancer journal for clinicians. 2017;67(2):122-37.

3. Siegel RL, Miller KD, Jemal A. Cancer statistics, 2018. CA: a cancer journal for clinicians. 2018;68(1):730 .

4. Siegel RL, Miller KD, Jemal A. Cancer statistics, 2019. CA: a cancer journal for clinicians. 2019;69(1):734 .

5. Chaturvedi AK, Engels EA, Pfeiffer RM, Hernandez BY, Xiao W, Kim E, et al. Human papillomavirus and rising oropharyngeal cancer incidence in the United States. Journal of clinical oncology : official journal of the American Society of Clinical Oncology. 2011;29(32):4294-301.

6. Santuray RT, Johnson DE, Grandis JR. New Therapies in Head and Neck Cancer. Trends in cancer. 2018;4(5):385-96.

7. Vermorken JB, Mesia R, Rivera F, Remenar E, Kawecki A, Rottey S, et al. Platinum-based chemotherapy plus cetuximab in head and neck cancer. The New England journal of medicine. 2008;359(11):1116-27.

8. Gillison ML, Trotti AM, Harris J, Eisbruch A, Harari PM, Adelstein DJ, et al. Radiotherapy plus cetuximab or cisplatin in human papillomavirus-positive oropharyngeal cancer (NRG Oncology RTOG 1016): a randomised, multicentre, non-inferiority trial. Lancet. 2019;393(10166):40-50.

9. Sanmamed MF, Chen L. A Paradigm Shift in Cancer Immunotherapy: From Enhancement to Normalization. Cell. 2018;175(2):313-26.

10. Chen J, Jiang CC, Jin L, Zhang XD. Regulation of PD-L1: a novel role of pro-survival signalling in cancer. Annals of oncology : official journal of the European Society for Medical Oncology. 2016;27(3):409-16.

11. Akinleye A, Rasool Z. Immune checkpoint inhibitors of PD-L1 as cancer therapeutics. Journal of hematology \& oncology. 2019;12(1):92. 
12. Galvis MM, Borges GA, Oliveira TB, Toledo IP, Castilho RM, Guerra ENS, et al. Immunotherapy improves efficacy and safety of patients with HPV positive and negative head and neck cancer: A systematic review and meta-analysis. Critical reviews in oncology/hematology. 2020;150:102966.

13. Wang BC, Cao RB, Fu C, Chen WB, Li PD, Lin GH, et al. The efficacy and safety of PD-1/PDL1 inhibitors in patients with recurrent or metastatic nasopharyngeal carcinoma: A systematic review and meta-analysis. Oral Oncol. 2020;104:104640.

14. Xu Y, Zhu G, Maroun CA, Wu IXY, Huang D, Seiwert TY, et al. Programmed Death-1/Programmed Death-Ligand 1-Axis Blockade in Recurrent or Metastatic Head and Neck Squamous Cell Carcinoma Stratified by Human Papillomavirus Status: A Systematic Review and Meta-Analysis. Frontiers in immunology. 2021;12:645170.

15. Moher D, Liberati A, Tetzlaff J, Altman DG. Preferred reporting items for systematic reviews and meta-analyses: the PRISMA statement. BMJ (Clinical research ed). 2009;339:b2535.

16. Mark Mitchell BMaTWea. "Engauge Digitizer Software.".http://markummitchellgithubio/engaugedigitizer. (accessed: January 8, 2021).

17. Tierney JF, Stewart LA, Ghersi D, Burdett S, Sydes MR. Practical methods for incorporating summary time-to-event data into meta-analysis. Trials. 2007;8:16.

18. Schünemann H BJ, Guyatt G, Oxman A, editors. GRADE handbook for grading quality of evidence and strength of recommendations. Updated October 2013. . The GRADE Working Group

http://guidelinedevelopmentorg/handbook2013. (accessed: February 19, 2021).

19. Balshem H, Helfand M, Schünemann HJ, Oxman AD, Kunz R, Brozek J, et al. GRADE guidelines: 3. Rating the quality of evidence. Journal of clinical epidemiology. 2011;64(4):401-6.

20. University M. GRADEpro GDT: GRADEpro Guideline Development Tool [Software]. http://gradeproorg/. 2020 (developed by Evidence Prime, Inc.).(accessed: February 19, 2021).

21. Ferris RL, Blumenschein G, Jr., Fayette J, Guigay J, Colevas AD, Licitra L, et al. Nivolumab for Recurrent Squamous-Cell Carcinoma of the Head and Neck. The New England journal of medicine. 2016;375(19):185667.

22. Burtness B, Harrington KJ, Greil R, Soulieres D, Tahara M, de Castro G, et al. Pembrolizumab alone or with chemotherapy versus cetuximab with chemotherapy for recurrent or metastatic squamous cell carcinoma of the head and neck (KEYNOTE-048): a randomised, open-label, phase 3 study. Lancet. 2019;394(10212):1915-28.

23. Cohen EEW, Soulières D, Le Tourneau C, Dinis J, Licitra L, Ahn MJ, et al. Pembrolizumab versus methotrexate, docetaxel, or cetuximab for recurrent or metastatic head-and-neck squamous cell carcinoma (KEYNOTE-040): a randomised, open-label, phase 3 study. The Lancet. 2019;393(10167):156-67.

24. Ferris RL, Haddad R, Even C, Tahara M, Dvorkin M, Ciuleanu TE, et al. Durvalumab with or without tremelimumab in patients with recurrent or metastatic head and neck squamous cell carcinoma: EAGLE, a randomized, open -label phase III study. Annals of Oncology. 2020;31(7):942-50.

25. Tao Y, Aupérin A, Sun X, Sire C, Martin L, Coutte A, et al. Avelumab-cetuximab-radiotherapy versus standards of care in locally advanced squamous-cell carcinoma of the head and neck: The safety phase of a randomised phase III trial GORTEC 2017-01 (REACH). European Journal of Cancer. 2020;141:21-9.

26. Seiwert TY, Burtness B, Mehra R, Weiss J, Berger R, Eder JP, et al. Safety and clinical activity of pembrolizumab for treatment of recurrent or metastatic squamous cell carcinoma of the head and neck (KEYNOTE-012): an open-label, multicentre, phase 1b trial. Lancet Oncology. 2016;17(7):956-65. 
27. Bauml J, Seiwert TY, Pfister DG, Worden F, Liu SV, Gilbert J, et al. Pembrolizumab for Platinumand Cetuximab-Refractory Head and Neck Cancer: Results From a Single-Arm, Phase II Study. Journal of clinical oncology : official journal of the American Society of Clinical Oncology. 2017;35(14):1542-9.

28. Colevas AD, Bahleda R, Braiteh F, Balmanoukian A, Brana I, Chau NG, et al. Safety and clinical activity of atezolizumab in head and neck cancer: results from a phase I trial. Annals of Oncology. 2018;29(11):224753.

29. Segal NH, Ou SHI, Balmanoukian A, Fury MG, Massarelli E, Brahmer JR, et al. Safety and efficacy of durvalumab in patients with head and neck squamous cell carcinoma: results from a phase I/II expansion cohort. European Journal of Cancer. 2019;109:154-61.

30. Siu LL, Even C, Mesia R, Remenar E, Daste A, Delord JP, et al. Safety and Efficacy of Durvalumab With or Without Tremelimumab in Patients With PD-L1-Low/Negative Recurrent or Metastatic HNSCC The Phase 2 CONDOR Randomized Clinical Trial. Jama Oncology. 2019;5(2):195-203.

31. Zandberg DP, Algazi AP, Jimeno A, Good JS, Fayette J, Bouganim N, et al. Durvalumab for recurrent or metastatic head and neck squamous cell carcinoma: Results from a single-arm, phase II study in patients with [?]25\% tumour cell PD-L1 expression who have progressed on platinum-based chemotherapy. European journal of cancer (Oxford, England : 1990). 2019;107:142-52.

32. Elbers JBW, Al-Mamgani A, Tesseslaar MET, van den Brekel MWM, Lange CAH, van der Wal JE, et al. Immuno-radiotherapy with cetuximab and avelumab for advanced stage head and neck squamous cell carcinoma: Results from a phase-I trial. Radiotherapy and Oncology. 2020;142:79-84.

33. Powell SF, Gold KA, Gitau MM, Sumey CJ, Lohr MM, McGraw SC, et al. Safety and Efficacy of Pembrolizumab With Chemoradiotherapy in Locally Advanced Head and Neck Squamous Cell Carcinoma: A Phase IB Study. Journal of Clinical Oncology. 2020;38(21):2427-+.

34. Weiss J, Sheth S, Deal AM, Olson JEG, Patel S, Hackman TG, et al. Concurrent Definitive Immunoradiotherapy for Patients with Stage III-IV Head and Neck Cancer and Cisplatin Contraindication. Clinical Cancer Research. 2020;26(16):4260-7.

35. Ang KK, Harris J, Wheeler R, Weber R, Rosenthal DI, Nguyen-Tan PF, et al. Human papillomavirus and survival of patients with oropharyngeal cancer. The New England journal of medicine. 2010;363(1):24-35.

36. Chow LQM. Head and Neck Cancer. The New England journal of medicine. 2020;382(1):60-72.

37. Ishida Y, Agata Y, Shibahara K, Honjo T. Induced expression of PD-1, a novel member of the immunoglobulin gene superfamily, upon programmed cell death. The EMBO journal. 1992;11(11):3887-95.

38. Freeman GJ, Long AJ, Iwai Y, Bourque K, Chernova T, Nishimura H, et al. Engagement of the PD-1 immunoinhibitory receptor by a novel B7 family member leads to negative regulation of lymphocyte activation. The Journal of experimental medicine. 2000;192(7):1027-34.

39. Latchman Y, Wood CR, Chernova T, Chaudhary D, Borde M, Chernova I, et al. PD-L2 is a second ligand for PD-1 and inhibits T cell activation. Nature immunology. 2001;2(3):261-8.

40. Yearley JH, Gibson C, Yu N, Moon C, Murphy E, Juco J, et al. PD-L2 Expression in Human Tumors: Relevance to Anti-PD-1 Therapy in Cancer. Clinical cancer research : an official journal of the American Association for Cancer Research. 2017;23(12):3158-67.

41. Ferris RL. Immunology and Immunotherapy of Head and Neck Cancer. Journal of clinical oncology : official journal of the American Society of Clinical Oncology. 2015;33(29):3293-304.

42. Essers PBM, van der Heijden M, Verhagen CVM, Ploeg EM, de Roest RH, Leemans CR, et al. Drug Sensitivity Prediction Models Reveal a Link between DNA Repair Defects and Poor Prognosis in HNSCC. Cancer Res. 2019;79(21):5597-611. 
43. Kobayashi K, Hisamatsu K, Suzui N, Hara A, Tomita H, Miyazaki T. A Review of HPV-Related Head and Neck Cancer. Journal of clinical medicine. 2018;7(9).

44. Cillo AR, Kurten CHL, Tabib T, Qi Z, Onkar S, Wang T, et al. Immune Landscape of Viral- and Carcinogen-Driven Head and Neck Cancer. Immunity. 2020;52(1):183-99.e9.

45. Kansy BA, Concha-Benavente F, Srivastava RM, Jie HB, Shayan G, Lei Y, et al. PD-1 Status in CD8(+) T Cells Associates with Survival and Anti-PD-1 Therapeutic Outcomes in Head and Neck Cancer. Cancer Res. 2017;77(22):6353-64.

46. Poeta ML, Manola J, Goldwasser MA, Forastiere A, Benoit N, Califano JA, et al. TP53 mutations and survival in squamous-cell carcinoma of the head and neck. The New England journal of medicine. 2007;357(25):2552-61.

47. Solomon B, Young RJ, Rischin D. Head and neck squamous cell carcinoma: Genomics and emerging biomarkers for immunomodulatory cancer treatments. Semin Cancer Biol. 2018;52(Pt 2):228-40.

48. Lenouvel D, Gonzalez-Moles M, Ruiz-Avila I, Gonzalez-Ruiz L, Gonzalez-Ruiz I, Ramos-Garcia P. Prognostic and clinicopathological significance of PD-L1 overexpression in oral squamous cell carcinoma: A systematic review and comprehensive meta-analysis. Oral Oncol. 2020;106:104722.

49. Theodoraki MN, Yerneni SS, Hoffmann TK, Gooding WE, Whiteside TL. Clinical Significance of PDL1(+) Exosomes in Plasma of Head and Neck Cancer Patients. Clinical cancer research : an official journal of the American Association for Cancer Research. 2018;24(4):896-905.

50. Strati A, Koutsodontis G, Papaxoinis G, Angelidis I, Zavridou M, Economopoulou P, et al. Prognostic significance of PD-L1 expression on circulating tumor cells in patients with head and neck squamous cell carcinoma. Annals of oncology : official journal of the European Society for Medical Oncology. 2017;28(8):1923-33.

51. Orlov S, Salari F, Kashat L, Walfish PG. Induction of painless thyroiditis in patients receiving programmed death 1 receptor immunotherapy for metastatic malignancies. The Journal of clinical endocrinology and metabolism. 2015;100(5):1738-41.

52. Byun DJ, Wolchok JD, Rosenberg LM, Girotra M. Cancer immunotherapy - immune checkpoint blockade and associated endocrinopathies. Nature reviews Endocrinology. 2017;13(4):195-207.

Table 1 Characteristics of Studies and Participants

Figure captions

Figure 1 Flow chart of study selection

Figure 2 Overall survival (a), and progression-free survival (b), PD-L1 positive related overall survival (c), and PD-L1 negative related overall survival (d)Figure 3 HPV related overall survival (a), objective response rate (b), and HPV related disease control rate (c)Figure 4 Any grade (a) and grade [?]3 (b) adverse events of PD-1/PD-L1inhibitors alone groups Figure 5 Individual analyses of any grade adverse events of PD-1/PD-L1 inhibitors alone groupsFigure 6 Individual analyses of grade [?]3 adverse events of PD-1/PD-L1 inhibitors alone groups

Footnotes: PD-1: programmed death 1 receptor; PD-L1: programmed death-ligand1; HPV: human papillomavirus; ECOG: Eastern Cooperative Oncology Group. KEYNOTE-048 (1) represents the study involved PD-1/PD-L1 inhibitors alone groups versus control groups; KEYNOTE-048 (2) represents the study involved PD-1/PD-L1 inhibitors with conventional therapy groups versus control groups. REACH (1) represents the control group as standard of care (cisplatin); REACH (2) represents the control group as standard of care (cetuximab).

PD-L1 status was characterized by PD-L1 combined positive score (CPS) calculated by the percentage of tumor cells, lymphocytes, and macrophages in the amount of tumor cells (Burtness et al., 2019; Cohen et 
al., 2019). PD-L1 was positive when PD-L1 CPS [?]1, otherwise PD-L1 was negative.

\section{Hosted file}

Table 1.docx available at https://authorea.com/users/419812/articles/526187-programmedcell-death-1-receptor-programmed-death-ligand1-pd-1-pd-11-inhibitors-alone-or-withconventional-therapy-versus-standard-of-care-for-head-and-neck-cancer-a-systematicreview-and-meta-analysis

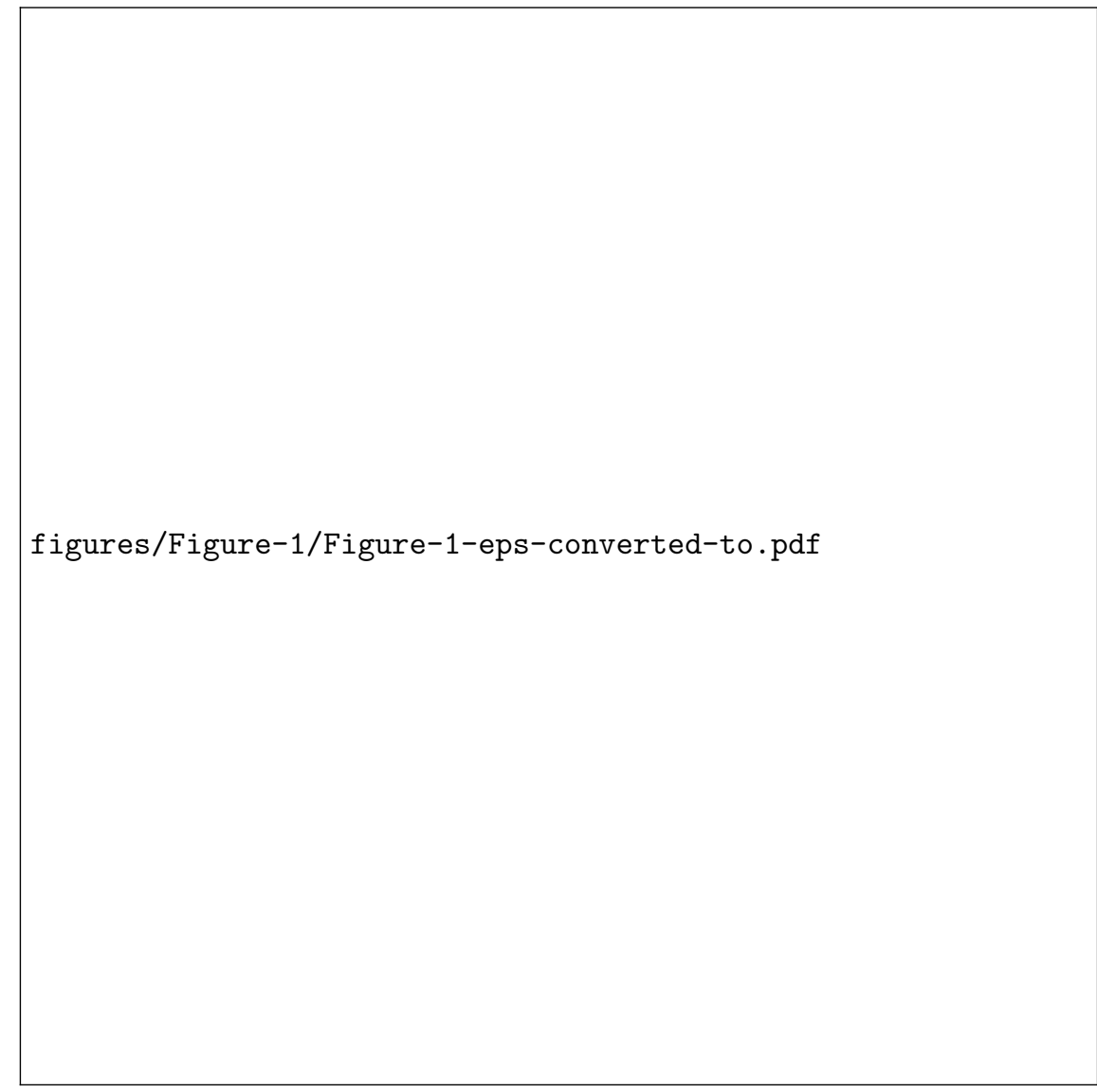


figures/Figure-2/Figure-2-eps-converted-to.pdf 
figures/Figure-3/Figure-3-eps-converted-to.pdf 
figures/Figure-4/Figure-4-eps-converted-to.pdf 
figures/Figure-5/Figure-5-eps-converted-to.pdf 
figures/Figure-6/Figure-6-eps-converted-to.pdf 